\title{
Grading of distal colorectal adenomas as predictors for proximal colonic neoplasia and choice of endoscope in population screening: experience from the Norwegian Colorectal Cancer Prevention study (NORCCAP)
}

\author{
G Gondal, T Grotmol, B Hofstad, M Bretthauer, T J Eide, G Hoff
}

Gut 2003;52:398-403

See end of article for authors' affiliations

Correspondence to: G Hoff, The Cancer Registry of Norway, Institute of

Population-based Cancer Research, Montebello, N-0310 Oslo, Norway; hofg@online.no

Accepted for publication: 27 November 2002

\begin{abstract}
Background and aims: The purpose of this study was to evaluate the utility of easily measured clinical variables at flexible sigmoidoscopy (FS) screening that might predict a proximal advanced neoplasm (PAN).

Methods: We studied 1833 subjects with biopsy verified adenomas at FS who subsequently underwent full colonoscopy.

Results: A total of $387(21 \%)$ subjects had proximal colonic neoplasms (PCN) and $85(5 \%)$ had PAN. In univariate comparison, the risk of PAN increased more than threefold in the presence of a distal adenoma measuring either $\geqslant 10 \mathrm{~mm}$ in diameter or containing villous components. Multiplicity of distal adenomas, severe dysplasia, or age $\geqslant 60$ years increased the risk of PAN more than twofold. In the multivariate model, the presence of a distal adenoma $\geqslant 10 \mathrm{~mm}$, villousness, and multiplicity maintained their significance as predictive variables for increased risk of proximal neoplasms, whereas sex and severe dysplasia lost their significance. By recommending colonoscopy only to individuals with multiple $(>1)$ adenomas or any high risk adenoma at FS, we would have reduced the number of colonoscopies by $1209(66 \%)$ but would have missed 32 (38\%) participants with PAN and $217(56 \%)$ with PCN. By using a $60 \mathrm{~cm}$ endoscope instead of an ordinary colonoscope at FS, nine (2\%) participants with advanced neoplasms, including three patients with cancer, would have been missed.

Conclusion: The present study supports the concept of defining "any adenoma" as a positive FS, qualifying for colonoscopy. We recommend the use of an ordinary colonoscope instead of a $60 \mathrm{~cm}$ sigmoidoscope for FS screening examinations.
\end{abstract}

\section{MATERIALS AND METHODS}

A detailed description of the study design has been published elsewhere.9 Briefly, the NORCCAP study is a prospective controlled study where 20780 men and women (1:1), aged 50-64 years, have been drawn randomly from the population registries of the City of Oslo and Telemark County and invited to a once only screening examination by means of FS or a combination of FS and faecal occult blood test.

Video colonoscopes were used in the screening centres both for FS and baseline colonoscopy (140 cm Olympus colonoscopes; Olympus Europa GmbH, Hamburg, Germany) with the exception of a satellite screening unit where, additionally, a $60 \mathrm{~cm}$ disposable endoscope system was used (Endosheath; Vision Sciences, Natick, Massachusetts, USA). In some participants repeated colonoscopies were required due to an inadequate initial examination or incomplete polypectomy. Only the first (baseline) colonoscopy, and only those where caecal intubation had been achieved, were included in the present analyses, thus excluding all patients with incomplete first attempt baseline colonoscopy. Depth of insertion at FS was limited by bowel cleansing, tolerance of the participant, and length of the endoscope available. Bowel cleansing for FS was limited to a $240 \mathrm{ml}$ sorbitol enema (Klyx; Ferring AS, Copenhagen, Denmark) administered at the screening centre before the examination. Re-examination after a repeat enema immediately or at a later date was performed when indicated, as judged by the endoscopist. Participants were given a printout of findings and level reached at FS, the latter expressed as the distance $(\mathrm{cm})$ from the anal verge with a straightened endoscope. Preparation for colonoscopy was performed using

Abbreviations: CRC, colorectal cancer; DCN, distal colorectal neoplasm; FS, flexible sigmoidoscopy; NORCCAP, Norwegian Colorectal Cancer Prevention study; PAN, proximal advanced neoplasm; $\mathrm{PCN}$, proximal colonic neoplasm; OR, odds ratio. 
a 4 litre polyethylene glycol solution (Laxabon (AstraZeneca AS, Oslo, Norway) or Klean-prep (Norgine Ltd, Middlesex, UK)). Sedation or antispasmodics were not used routinely at FS or colonoscopy. Biopsy samples were taken from all polyps visualised at FS. All polyps seen at colonoscopy were removed using cold or hot biopsy forceps (minute lesions only) or diathermy snare.

All neoplasms found at FS were defined as DCN irrespective of depth of endoscope insertion, whereas neoplastic lesions found at colonoscopy proximal to the level reached at FS were defined as PCN. Similarly, PAN was defined as high risk adenoma or carcinoma proximal to the level reached at FS. Patients with ulcerating or stricturing tumours discovered at FS were excluded from the present study of PCN in relation to DCN as these patients were already destined for colonoscopy as part of the preoperative workup.

Histological classification and grading of dysplasia in adenomas followed the WHO guidelines with some modifications. ${ }^{9}$ A diagnosis of adenoma at FS was entirely based on histopathological examination of the biopsy specimens. The findings in the polypectomy specimen at colonoscopy were backdated to characterise FS adenomas in greater detail (dysplasia/villous components). Data recorded for each polyp included size ( $\mathrm{mm}$ diameter) and localisation. Polyp size was determined by comparison with an open or closed biopsy forceps held against the lesion before taking a biopsy and in some cases of pedunculated polyps by direct measurement after polypectomy and retrieval. Polyp localisation was estimated using endoscopic landmarks in addition to distance from the anal verge measured at withdrawal of the straightened endoscope-that is, straightening to the point where the visual field starts to slip away.

Reproducibility of localisation of the most proximal index adenoma at FS was crucial to differentiate this lesion from the most distal of any PCN found at colonoscopy. Level estimates differing $<5 \mathrm{~cm}$ were accepted up to the $30 \mathrm{~cm}$ level and $<10$ $\mathrm{cm}$ beyond this to define an FS adenoma as recognised at colonoscopy. Likewise, a difference of $\pm 3 \mathrm{~mm}$ in diameter was accepted when adenoma size was $<10 \mathrm{~mm}$ but a $\pm 5 \mathrm{~mm}$ difference in diameter was accepted in the case of adenomas $\geqslant 10$ $\mathrm{mm}$. In the case of a solitary adenoma within a bowel segment, a larger difference was accepted provided that other criteria (histology) indicated recovery of a lesion already identified at FS. A reduction in diameter of up to $4 \mathrm{~mm}$ was accepted if biopsies had been taken at FS. A positive FS was defined as a finding of any biopsy verified adenoma, irrespective of size (index adenoma). In cases of multiple index adenomas with different characteristics or multiple characteristics in a solitary adenoma, analyses were performed separately for the characteristics size, degree of dysplasia (mild, moderate, or severe), and villousness. Adenomas with mild or moderate dysplasia were pooled into one group in the statistical analysis. A high risk adenoma was defined as an adenoma measuring $\geqslant 10 \mathrm{~mm}$ in diameter and/or with villous components ( $>20 \%$ of the lesion) and/or showing severe dysplasia. The same criteria were used to define advanced lesions which additionally included cases of adenocarcinoma.

\section{Ethics}

The regional ethics committee and the National Institute of Data Inspection approved the study protocol. Written informed consent was obtained.

\section{Statistical methods}

Logistic regression analysis was used to determine which features of the index adenoma(s) were most important for predicting the presence of PCN and PAN detected at subsequent colonoscopy. The association between the various independent variables and the presence of PCN and PAN was expressed as odds ratios (ORs) with 95\% confidence interval
(CI) and estimated by logistic regression analysis applying the statistical software SPSS 11.0 (SPSS, Chicago, Illinois, USA). Explanatory variables included the following characteristics of the index adenoma(s): multiplicity, diameter of the largest one, most severe dysplasia, and villousness. These variables were dichotomised to facilitate interpretation of the ORs. As the frequency of adenomas is related to sex (more frequent in men) and age (increasing frequency with age), the variables sex and age were included as covariates in the model. ${ }^{10}$

A univariate analysis was carried out initially for each explanatory variable (thus providing crude ORs), and subsequently all variables were included in a multivariate logistic regression analysis (providing adjusted ORs). Focussing on PAN, the association between the features of the index adenoma(s) and PCN was analysed only in a univariate model to restrict the volume of data. For age, a test for trend over the three age categories (50-54,55-59, and 60-64 years) was performed by treating age category as a continuous variable in the logistic regression model. A p value $<0.05$ - that is, $95 \%$ CI not encompassing the value 1.00 -was regarded as statistically significant.

\section{RESULTS}

A total of 2154 (17\%) of 12960 screenees had neoplasms at FS: $1293(21 \%)$ men and 861 (13\%) women. Eighty five patients who declined a baseline colonoscopy were excluded, together with nine subjects who were examined to the caecum during FS and six individuals who had colonoscopy performed by external endoscopists. A total of $193(9 \%)$ patients in whom caecal intubation was not achieved during the first colonoscopy (119 (14\%) women and $74(6 \%)$ men) and $28(0.2 \%)$ patients with cancer discovered at FS were also excluded from the present analyses. Thus the study population consisted of 1833 individuals (1131 (62\%) males and 702 (38\%) females). Mean age was 57 (SD 3.7) years. For FS, bowel cleansing was judged to be excellent in $1469(80 \%)$, adequate in 160 (9\%), poor in $203(11 \%)$, and data missing in one. Mean depth of insertion at FS was 48 (SD 15.1) cm measured with a straightened endoscope at withdrawal. Examination beyond the splenic flexure was judged by the individual endoscopist to have been achieved in $113(6 \%)$ patients at FS. The average time from FS to baseline colonoscopy was 58 days. Only 43 $(2 \%)$ of 1833 screenees postponed a recommended colonoscopy for more than six months. Bowel cleansing for colonoscopy was considered excellent in 1433 (78\%), adequate in $292(16 \%)$, poor in $102(6 \%)$, and data missing in six. For separate analysis of the benefit of using a $140 \mathrm{~cm}$ versus a 60 $\mathrm{cm}$ endoscope, the nine patients examined to the caecum at FS were included, thus comprising 1842 patients for this analysis.

\section{Characteristics of index adenomas}

Histological characteristics of index adenomas found at FS are shown in table $1: 341(15 \%)$ index adenomas were $\geqslant 10 \mathrm{~mm}$ in diameter. In total, $454(20 \%)$ adenomas at FS were high risk index adenomas. Index adenomas with villous components were found in $157(9 \%)$, severe dysplasia in $112(6 \%)$, and a solitary adenoma in 1502 (82\%) screenees (table 2).

\section{Characteristics of proximal colonic neoplasms}

\section{Any proximal colonic neoplasm (PCN)}

These data are shown in table 2 . We found that $387(21 \%)$ of 1833 participants with DCN had synchronous PCN at colonoscopy. PCN were detected in $72(14 \%)$ participants aged $50-54$ years, $173(22 \%)$ at 55-59 years of age, and $142(27 \%)$ at age 60-64 years ( $\mathrm{p}$ for trend=0.01). A total of $117(28 \%)$ individuals with high risk distal adenoma(s) had PCN compared with $270(19 \%)$ participants with low risk distal adenomas (OR 1.66, 95\% CI 1.29-2.14; $\mathrm{p}=0.01$ ). 
Table 1 Characteristics of adenomas identified at flexible sigmoidoscopy screening in 1833 individuals (per cent)

\begin{tabular}{|c|c|c|c|c|c|}
\hline \multicolumn{2}{|l|}{ Histological features } & \multicolumn{3}{|l|}{ Diameter } & \multirow[b]{2}{*}{ Total } \\
\hline Type of adenoma & Dysplasia & $<5 \mathrm{~mm}$ & $5-9 \mathrm{~mm}$ & $\geqslant 10 \mathrm{~mm}$ & \\
\hline Tubular & Mild or moderate & $1175(58)$ & $636(32)$ & 206 (10) & $2017(100)$ \\
\hline Tubular & Severe & 11 (13) & 31 (37) & 41 (50) & 83 (100) \\
\hline Villous components & Mild or moderate & 12 (9) & $54(41)$ & $65(50)$ & 131 (100) \\
\hline Villous components & Severe & 1 (3) & 4 (12) & 29 (85) & $34(100)$ \\
\hline Total & & 1199 (53) & 725 (32) & 341 (15) & 2265 (100) \\
\hline
\end{tabular}

Table 2 Prevalence of proximal neoplasia (PCN) and proximal advanced neoplasia (PAN) at full colonoscopy according to a finding of distal colorectal adenoma (index adenoma) at flexible sigmoidoscopy (FS) among 1833 participants

\begin{tabular}{lcrl}
\hline $\begin{array}{l}\text { Adenoma characteristics } \\
\text { in individuals with index } \\
\text { adenomas at FS }\end{array}$ & $\begin{array}{l}\text { No of } \\
\text { subjects } \\
(n=1833)\end{array}$ & $\begin{array}{l}\text { PCN } \\
(n=387)\end{array}$ & $\begin{array}{l}\text { PAN } \\
(n=85)\end{array}$ \\
\hline Adenoma <10 mm & 1510 & $295(20)$ & $50(3)$ \\
Adenoma $\geqslant 10 \mathrm{~mm}$ & 323 & $92(28)$ & $35(11)$ \\
Mild/moderate dysplasia & 1721 & $353(21)$ & $75(4)$ \\
Severe dysplasia & 112 & $34(30)$ & $10(9)$ \\
No villous components & 1676 & $347(21)$ & $66(4)$ \\
Villous components & 157 & $40(25)$ & $19(12)$ \\
Solitary adenoma & 1502 & $285(19)$ & $55(4)$ \\
Multiple adenomas & 331 & $102(31)$ & $30(9)$ \\
Female & 702 & $129(18)$ & $23(3)$ \\
Male & 1131 & $258(23)$ & $62(6)$ \\
Age 50-54 years & 502 & $72(14)$ & $13(3)$ \\
$\quad 55-59$ years & 802 & $173(22)$ & $35(4)$ \\
$\quad 60-64$ years & 529 & $142(27)$ & $37(7)$ \\
Distal low risk adenoma & 1417 & $270(19)$ & $43(3)$ \\
Distal high risk adenoma & 416 & $117(28)$ & $42(10)$ \\
\hline
\end{tabular}

Values in parentheses indicate percentages of individuals with the given characteristic relative to the total number of individuals with an index adenoma.

Proximal advanced neoplasms (PAN)

These data are summarised in tables 2 and 3. Of 323 participants with an index adenoma $\geqslant 10 \mathrm{~mm}, 35(11 \%)$ had PAN compared with 50 (3\%) of 1510 with an index adenoma $<10 \mathrm{~mm}$ (OR 3.56; $\mathrm{p}=0.01$ ). Ten $(9 \%)$ of 112 individuals with a severely dysplastic index adenoma had PAN compared with $75(4 \%)$ of 1721 with an index adenoma showing only mild/moderate dysplasia (OR 2.15; $\mathrm{p}=0.03$ ). Of 157 participants with an index adenoma containing villous components, $19(12 \%)$ had a PAN compared with $66(4 \%)$ of 1676 with an index adenoma with only tubular components (OR 3.38; $\mathrm{p}=0.01)$. Thirty $(9 \%)$ of 331 participants with multiple index adenomas had PAN compared with 55 (4\%) of 1502 individuals with a solitary index adenoma (OR 2.62; $\mathrm{p}=0.01$ ). Sixty two $(6 \%)$ of 1131 males had PAN compared with $23(3 \%)$ of 702 females (OR 1.71; $\mathrm{p}=0.03$ ). Thirteen $(3 \%)$ of 502 participants aged 50-54 years had PAN compared with $35(4 \%)$ of 802 individuals aged $55-59$ years and 37 (7\%) of 529 participants aged 60-64 years (OR 1.67; $\mathrm{p}$ for trend $=0.01$ ).

All of the associations listed above pertained to a univariate logistic regression analysis and were statistically significant. All six variables were subsequently included in a multivariate analysis where "adenoma size", "villousness", "adenoma frequency", and "age" remained significant. These variables are thus independent and significant predictors of PAN. The variables "most severe dysplasia" and "gender" lost their significance. By excluding variables one at a time, the variable "most severe dysplasia" lost its significance in the multivariate analysis due to its intercorrelation with "adenoma size", "vil- lousness", and "frequency". The variables "gender" and "age" were included as covariates in the model and their interrelation with the other variables was not explored any further.

\section{Threshold at FS screening for referral to colonoscopy}

Multiple (>1) or high risk index adenoma(s)

If we had recommended colonoscopy only to participants with multiple $(>1)$ or a high risk index adenoma(s), we would have reduced the number of colonoscopies by 1209 (66\%) from 1833. We would have missed $32(38 \%)$ of 85 PAN, including one of six proximal colon cancers, and 217 (56\%) of 387 PCN. A similar type of calculation was performed for each of the three age categories separately, and the results are given in table 4. There was a trend towards a higher proportion of saved colonoscopies in the youngest age group combined with less severe loss of proximal lesions.

Three or more adenomas or any high risk index adenoma If we had recommended colonoscopy only to individuals with three or more adenomas or any high risk adenoma for all ages combined, we would have saved $1373(75 \%)$ of 1833 colonoscopies but we would have missed 39 (46\%) of 85 patients with PAN, including one of six with proximal colon cancer, and 251 (65\%) of 387 PCN. A similar type of calculation was performed for each of the three age categories separately, and the results are given in table 4 . As for the previous simulation, there was a trend towards a higher proportion of saved colonoscopies in the youngest age group.

\section{Comparison of "real life FS" in NORCCAP versus}

"surrogate FS" (first $60 \mathrm{~cm}$ of a full length colonoscope) A total of $316(17 \%)$ of 1842 patients were examined beyond $60 \mathrm{~cm}$ (endoscope insertion) at FS. If we had used only a 60 $\mathrm{cm}$ endoscope, 56 (3\%) of 1842 individuals with index adenomas would have been diagnosed as adenoma free (false negative screening test). These missed cases would have included 5 ( $1 \%$ ) of 416 with a distal high risk adenoma localised beyond $60 \mathrm{~cm}$. With no index lesion to trigger a colonoscopy, we would subsequently have missed 1 ( $1 \%$ ) of 85 cases with PAN and 10 (3\%) of 387 with PCN. In total, $9(2 \%)$ of 487 patients with advanced neoplasms, including three patients with proximal colon cancers, would have been misclassified as having no neoplasia. One of these three cancer cases had a past history of benign polyps and had therefore been given an appointment for colonoscopy elsewhere at the same time. This was instead performed as a screening procedure by one of our endoscopists.

\section{DISCUSSION}

Several endoscopic studies on PCN have addressed the predictive value of distal index lesions. Some have evaluated "surrogate FS" (the first part (for example, $60 \mathrm{~cm}$ ) of a colonoscopic examination) versus full colonoscopy with bowel cleansing preparation for full colonoscopy. ${ }^{11-13}$ The latter study also accepted inclusion of additional findings at repeat colonoscopy if baseline colonoscopy was incomplete, taking advantage of increased pick up rates for polyps during repeat 
Table 3 Crude and adjusted odds ratio (OR) for having proximal advanced neoplasia diagnosed at colonoscopy, depending on the characteristics of distal colorectal adenomas (index adenoma) diagnosed at flexible sigmoidocopy (FS) among 1833 participants

\begin{tabular}{|c|c|c|c|c|c|}
\hline Variable at FS screening & $\begin{array}{l}\text { No of } \\
\text { individuals }\end{array}$ & Crude OR & $95 \% \mathrm{Cl}$ & Adjusted OR & $95 \% \mathrm{Cl}$ \\
\hline \multicolumn{6}{|c|}{ Diameter of largest index adenoma } \\
\hline Adenoma $<10 \mathrm{~mm}$ & 1510 & Referent & & 1.0 & \\
\hline Adenoma $\geqslant 10 \mathrm{~mm}$ & 323 & 3.56 & $2.27-5.58$ & 2.54 & $1.51-4.26$ \\
\hline \multicolumn{6}{|c|}{ Most severe dysplasia of index adenoma } \\
\hline Mild/moderate dysplasia & 1721 & Referent & & 1.0 & \\
\hline Severe dysplasia & 112 & 2.15 & $1.08-4.29$ & 1.06 & $0.49-2.27$ \\
\hline \multicolumn{6}{|l|}{ Villousness of index adenoma } \\
\hline No villous components & 1676 & Referent & & 1.0 & \\
\hline Villous components & 157 & 3.38 & $1.97-5.80$ & 1.85 & $1.00-3.39$ \\
\hline \multicolumn{6}{|l|}{ Multiplicity } \\
\hline Solitary index adenoma & 1502 & Referent & & 1.0 & \\
\hline Multiple index adenoma & 331 & 2.62 & $1.65-4.16$ & 1.95 & $1.20-3.17$ \\
\hline \multicolumn{6}{|l|}{ Sex } \\
\hline Female & 702 & Referent & & 1.0 & \\
\hline Male & 1131 & 1.71 & $1.05-2.79$ & 1.59 & $0.96-2.62$ \\
\hline \multicolumn{6}{|l|}{ Age $(y)$} \\
\hline $50-54$ & 502 & Referent & & 1.0 & \\
\hline $55-59$ & 802 & 1.72 & $0.90-3.27$ & 1.53 & $0.80-2.95$ \\
\hline $\begin{array}{c}60-64 \\
p \text { for trend }\end{array}$ & 529 & $\begin{array}{r}2.82 \\
<0.01\end{array}$ & $1.48-5.38$ & $\begin{array}{c}2.55 \\
<0.003\end{array}$ & $1.32-4.91$ \\
\hline
\end{tabular}

Table 4 Cost benefit of two baseline colonoscopy scenarios expressed by examinations saved and lesions missed (per cent) among 1833 participants

\begin{tabular}{|c|c|c|c|c|}
\hline $\begin{array}{l}\text { Thresholds for baseline } \\
\text { colonoscopy after FS } \\
\text { screening }\end{array}$ & $\begin{array}{l}\text { No of } \\
\text { colonoscopies } \\
\text { saved }(\%)^{*}\end{array}$ & $\begin{array}{l}\text { No of missed } \\
\text { cases of PCN } \\
(\%)^{*}\end{array}$ & $\begin{array}{l}\text { No of missed } \\
\text { cases of PAN } \\
(\%)^{*}\end{array}$ & $\begin{array}{l}\text { No of missed cases } \\
\text { of proximal colonic } \\
\text { cancer }(\%)^{*}\end{array}$ \\
\hline \multicolumn{5}{|c|}{ Multiple (>1) or high risk index adenoma } \\
\hline Age $50-54$ years & $362 / 502$ (72) & $39 / 72(53)$ & $3 / 13$ (23) & $0 / 1$ \\
\hline Age $55-59$ years & $512 / 802(64)$ & $99 / 173$ (57) & $14 / 35(40)$ & $0 / 2$ \\
\hline Age $60-64$ years & $335 / 529(63)$ & $79 / 142(56)$ & $15 / 37$ (40) & $1 / 3(33)$ \\
\hline Total & $1209 / 1833(66)$ & $217 / 387(56)$ & $32 / 85(38)$ & $1 / 6(17)$ \\
\hline \multicolumn{5}{|c|}{ Three or more adenomas or any high risk index adenomas } \\
\hline Age $50-54$ years & $401 / 502$ (80) & $44 / 72(61)$ & $4 / 13$ (31) & $0 / 1$ \\
\hline Age $55-59$ years & $589 / 802(73)$ & $114 / 173(66)$ & $18 / 35(51)$ & $0 / 1$ \\
\hline Age $60-64$ years & $383 / 529(72)$ & $93 / 142$ (65) & $17 / 37$ (46) & $1 / 3(33)$ \\
\hline Total & $1373 / 1833$ (75) & $251 / 387$ (65) & $39 / 85$ (46) & $1 / 6(17)$ \\
\hline
\end{tabular}

examinations. In the present study we wished to evaluate real life "once only" FS screening using a simple bowel cleansing procedure followed by a once only full colonoscopy, when indicated, according to the protocol. Others have also used ordinary colonoscopes for FS screening..$^{14}$ It is rarely stated whether insertion level and polyp localisation level (in $\mathrm{cm}$ from the anal verge) are measured at maximal insertion or with maximal straightening of the endoscope. This has methodological implications as level estimates differ during insertion and withdrawal beyond $30 \mathrm{~cm} .{ }^{15}$ This may also influence results on distal versus proximal findings at two separate examinations. In the present study, level estimates were standardised with the endoscope in a straightened position. This may explain why the average level reached at FS was shorter $(48 \mathrm{~cm})$ than in other studies using enema for cleansing (56 and $58 \mathrm{~cm}$, respectively ${ }^{4}{ }^{14}$ ).

In our multivariate analysis the variables "index adenoma $\geqslant 10 \mathrm{~mm}^{\prime}$ " "villousness", and "multiplicity" remained independent predictive factors for increased risk of PAN whereas "gender" and "severe dysplasia" lost their significance. A study in a population of mixed symptomatic and asymptomatic patients showed that size and histology of a distal adenoma at FS were predictors of PAN whereas the number of distal adenomas was not. ${ }^{16}$ Another study where selected cases of incomplete polypectomies (largely hyperplastic polyps) comprised a large proportion of those subjected to colonoscopy showed that histology and number of distal adenomas were important predictors of PAN while the size of distal adenomas was not. This study defined additional new lesions at colonoscopy as distal index lesions if situated within the FS range, again taking advantage of additional findings at repeat examination but giving an overestimation of findings at real life screening. ${ }^{17}$ The availability of a satisfactory colonoscopy service is restricted in most countries, and cost/benefit considerations of different thresholds for colonoscopy are most welcome. In a once only FS screening concept, even low risk lesions may be of importance. Some of these may develop through stages of high risk lesions to cancer during a time span estimated to be 10-15 years rather than the 3-4 years expected for high risk adenomas. ${ }^{18}$ Only long term follow up of comparative studies using different thresholds for proceeding from FS to baseline colonoscopy will render information as to what is the most feasible and beneficial working definition of a positive FS qualifying for full colonoscopy. We now have a situation when that may be possible as both the ongoing Italian $^{19}$ and British Flexi-Scope trials ${ }^{20}$ use high risk adenoma 
or $\geqslant 3$ adenomas as thresholds for colonoscopy in contrast with "any adenoma" in the present study. A number of experts have advocated limiting colonoscopy to individuals with distal advanced adenomas found at FS. ${ }^{61721}$ Atkin et al studied 1618 patients in a case control study, on average 14 years after removal of high risk or low risk adenomas at rigid sigmoidoscopy without subsequent endoscopy surveillance. Their study showed a higher than expected risk of subsequent colon cancer confined to the group with high risk rectal adenomas. ${ }^{21}$ In a smaller study of 226 patients, individuals with a high risk adenoma at FS were 15 times more likely to have a proximal high risk adenoma than individuals without a distal high risk adenoma. ${ }^{16}$ In the present study, by recommending colonoscopy to individuals with multiple index adenomas or any high risk adenoma, we would have missed $32(38 \%)$ patients with PAN, including one case of proximal cancer.

In the British Flexi-Scope trial, 40674 individuals have been examined by means of FS at age 55-64 years. ${ }^{20} \mathrm{~A}$ total of 2131 $(5 \%)$ individuals were referred for colonoscopy compared with $2154(17 \%)$ in our study. The burden of colonoscopies is quite considerable when applying the low NORCCAP threshold for colonoscopies. By applying the British Flexi- Scope trial criteria for a positive FS in age group 55-64 years, we would have saved 972 (73\%) of our colonoscopies, missed 35 (49\%) cases of PAN, including one cancer, and 207 (66\%) of our cases with PCN. At the other end of the scale, we do not know the extent of missed PAN using FS screening with a low threshold for colonoscopy compared with a primary "full distance" colonoscopy screening examination. Lieberman and Weiss studied 2885 asymptomatic subjects aged 50-75 years who provided stool specimens and underwent a complete colonoscopy. ${ }^{11}$ Examination of the first $60 \mathrm{~cm}$ of the rectum and sigmoid colon during colonoscopy was used as a surrogate for FS, and "any adenoma at FS" was defined as a positive screening test. They concluded that once only FS would miss about $30 \%$ of patients with advanced proximal neoplasms. Differences between their study and ours were a mean age of 63 versus 57 years of age and a dominant male sex of $97 \%$ in their study. Similarly, Lemmel et al estimated that approximately $30 \%$ of all colorectal cancers would be missed at FS screening as that proportion was found to be beyond the reach of FS with no detectable index adenoma in their material having a mean age of 69.5 years. ${ }^{22}$ With increasing age there is a right sided shift of colonic neoplasms possibly making FS screening less rewarding at ages above $60-65$ years. ${ }^{23}$

Using a colonoscope in the present study has taken some advantage of the fact that even a single enema may allow endoscopy beyond the reach of an ordinary flexible sigmoidoscope in a large proportion of screenees $(316(17 \%)$ in our hands). FS was performed with the screenee lying in the left lateral position allowing examination by merely pulling down the patient's clothes. This, together with limited time at FS, did not permit taking full advantage of using a colonoscope for the examination. In our study only $6 \%$ of participants were judged to have been examined proximal to the splenic flexure. The true anatomical extent of the examination may be difficult to assess as endoscopic landmarks have been shown to be very unreliable. ${ }^{24}$ Having eliminated length of the endoscope as a limiting factor, we were essentially left with degree of bowel cleansing and patient tolerance as end points for each examination. If we had used a $60 \mathrm{~cm}$ endoscope, $9(2 \%)$ of our participants with advanced neoplasms, including three cases of proximal colon cancer, would have been misclassified as neoplasia free. As pointed out, one of these carcinomas would have been diagnosed elsewhere as the patient was due for a surveillance appointment. An important fringe benefit of using a full length colonoscope for FS screening examinations may be to encourage achieving the technical ability to perform painless examinations beyond the descending/sigmoid junction and further acceptance for unsedated colonoscopy. ${ }^{23}$

A few studies have looked at the prevalence of PCN in relation to the presence or absence of low risk adenomas at FS. ${ }^{4-8}$
These studies have shown that the prevalence of PCN (mostly diminutive) is about the same, irrespective of the presence or absence of adenomas at FS. Most studies shedding some light on this have been small, with selected participants, and most of the participants were older than 60 years of age. It has been estimated that adenomas less than $5 \mathrm{~mm}$ tend to grow but when reaching 5-9 $\mathrm{mm}$ most show a tendency to regress during a three year period of follow up, ${ }^{25}$ consistent with the view that most adenomas do not progress to clinical relevance. In a study of 226 patients with radiologically detected polyps $>10$ mm left untreated, $2.5 \%$ of patients had developed cancer in that particular bowel segment after five years and $24 \%$ after 20 years, supporting the prevailing view that large polyps should not be ignored ${ }^{26}$ As most small and even large adenomas may regress, we do not know the real importance of differences in thresholds for colonoscopy after FS.

In addition to the characteristics of index adenomas, we found that age was an important predictor for PAN. The risk of PAN increased on average $67 \%$ for each five year age increase between 50 and 64 years. Few comparable studies are available but in a study of colonoscopic screening of 1994 asymptomatic subjects who were $50-80$ years old, it was found that each five year increase in age raised the risk of colonic neoplasms by $32 \% .{ }^{12}$ Differences in reported risk of PAN in various studies may to a large extent be due to differences in age groups, as reported in an autopsy study where the age dependent adenoma prevalence rate increased towards a peak at 50-70 years of age with a flattening of the curve thereafter. ${ }^{10}$

In conclusion, baseline findings in the ongoing NORCCAP study support the concept of defining detection of any adenoma as a positive FS qualifying for full colonoscopy. The long term effect of differences in the definition of a positive FS can only be assessed by follow up results of ongoing studies having different thresholds for colonoscopy. The present study indicates that the full diagnostic benefit of unsedated FS with a simple enema for cleansing may not be obtained when using only a $60 \mathrm{~cm}$ flexible endoscope.

\section{ACKNOWLEDGEMENTS}

We owe thanks to the Norwegian Cancer Society and the Ministry of Health and Social Affairs for financial support and to the Norwegian Gastrointestinal Cancer Group (NGICG) for initiating the study.

\section{Authors' affiliations}

G Gondal, T Grotmol, M Bretthauer, G Hoff, The Cancer Registry of Norway, Institute of Population-based Cancer Research, Montebello, Oslo, Norway

B Hofstad, Department of Medicine, Ullevaal Hospital, Oslo, Norway T J Eide, Department of Pathology, Norwegian National Hospital, Oslo, Norway

\section{REFERENCES}

1 Selby JV, Friedman GD, Quesenberry CP, et al. A case-control study of screening sigmoidoscopy and mortality from colorectal cancer. N EnglJ Med 1992:326:653-7.

2 Atkin WS. Flexible sigmoidoscopy as a mass screening tool. Eur J Gastroenterol Hepatol 1998;10:219-23.

3 Thiis-Evensen E, Hoff GS, Sauar J, Langmark F, et al. Population-based surveillance by colonoscopy: Effect on the incidence of colorectal cancer. Scand I Gastroenterol 1999:34:414-20.

4 Foutch PG, Mai H, Pardy K, et al. Flexible sigmoidoscopy may be ineffective for secondary prevention of colorectal cancer in asymptomatic, average-risk men. Dig Dis Sci 1991;36:924-8.

5 Achkar E, Carey W. Small polyps found during fiberoptic sigmoidoscopy in asymptomatic patients. Ann Intern Med 1988:109:880-3.

6 Schoen RE, Corle D, Cranston L, et al. Is colonoscopy needed for the nonadvanced adenoma found on sigmoidoscopy? Gastroenterology 1998; 115:533-41

7 Kadakia SC, Wrobleski CS, Kadakia AS, et al. Prevalence of proximal colonic polyps in average-risk asymptomatic patients with negative fecal occult blood tests and flexible sigmoidoscopy. Gastrointest Endosc 1996:44:112-17

8 Rex DR, Lehman GA, Hawes RH, et al. Screening colonoscopy in asymptomatic average-risk persons with negative fecal occult blood tests. Gastroenterology 1991;100:64-7. 
9 Bretthauer M, Gondal G, Larsen IK, et al. Design, organization and management of a controlled population screening study for detection of colorectal neoplasia. Scand J Gastroenterol 2002;37:568-73.

10 Vatn MH, Stalsberg H. The prevalence of polyps of the large intestine in Oslo: an autopsy study. Cancer 1982;49:819-25

11 Lieberman DA, Weiss DG. One-time screening for colorectal cancer with combined fecal occult-blood testing and examination of the distal colon. N Engl J Med 2001;345:555-60.

12 Imperiale TF, Wagner DR, Lin CY, et al. Risk of advanced proxima neoplasms in asymptomatic adults according to the distal colorectal findings. N Engl J Med 2000;343:169-74.

13 Lieberman DA, Weiss DG, Bond JH, et al. Use of colonoscopy to screen asymptomatic adults for colorectal cancer. $N$ Engl J Med 2000;343: 162-8

14 Collett JA, Olynk JK, Platell CF. Flexible sigmoidoscopy screening for colorectal cancer in average-risk people: update of a community-based project. Med J Aust 2000;173:463-6.

15 Hoff G, Vatn M. Epidemiology of polyps in the rectum and sigmoid colon. Endoscopic evaluation of size and localization of polyps. Scand J Gastroenterol 1985;20:356-60.

16 Zarchy TM, Ershoff D. Do characteristics of adenomas on flexible sigmoidoscopy predict advanced lesions on baseline coloscopy? Gastroenterology 1994;106:1501-4.

17 Levin TR, Palitz A, Grossman S, et al. Predicting advanced proximal colonic neoplasia with screening sigmoidoscopy. JAMA 1999;281:1611-17.
18 Eide TJ. Risk of colorectal cancer in adenoma-bearing individuals within a defined population. Int J Cancer 1986:38:173-6.

19 Senore C, Segnan N, Rossini FP, et al. Screening for colorectal cancer by once only sigmoidoscopy. A feasibility study in Turin, Italy. J Med Screen 1996;3:72-8.

20 Atkin WS, Cook CF, Cuzick J, et al. Single flexible sigmoidoscopy screening to prevent colorectal cancer: baseline findings of a UK multicentre randomised trial. Lancet 2002;359:1291-8.

21 Atkin WS, Morson BC, Cuzick J. Long-term risk of colorectal cancer after excision of rectosigmoid adenomas. N Engl J Med 1992;326:658-62.

22 Lemmel GT, Haseman JH, Rex DK, et al. Neoplasia distal to the splenic flexure in patients with proximal colon cancer. Gastrointest Endosc 1996:44:109-11.

23 Thiis-Evensen E, Hoff GS, Sauar J, et al. Flexible sigmoidoscopy or colonoscopy as a screening modality for colorectal adenomas in older age groups? Findings in a cohort of the normal population aged 63-72 years. Gut 1999;45:834-9.

24 Painter J, Saunders DB, Bell GD, et al. Depth of insertion at flexible sigmoidoscopy: implications for colorectal cancer screening and instrument design. Endoscopy 1999:31:227-31.

25 Hofstad B, Vatn MH, Andersen SN, et al. Growth of colorectal polyps: redetection and evaluation of unresected polyps for a period of three years. Gut 1996;39:449-56

26 Stryker SJ, Wolff BG, Culp CE, et al. Natural history of untreated colonic polyps. Gastroenterology 1987;93:1009-13.

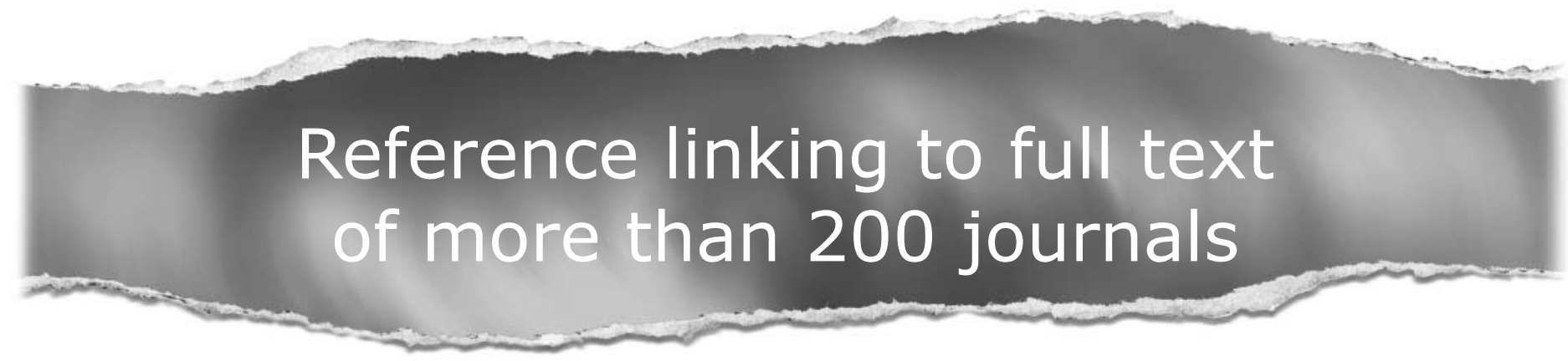

\section{Toll free links}

You can access the FULL TEXT of articles cited in Gut online if the citation is to one of the more than 200 journals hosted by HighWire (http://highwire.stanford.edu) without a subscription to that journal. There are also direct links from references to the Medline abstract for other titles.

\section{www.gutjnl.com}

